\title{
Spinodal Instability in the Quark-Gluon Plasma
}

\author{
C. E. Aguiar, E. S. Fraga, and T. Kodama \\ Instituto de Física, Universidade Federal do Rio de Janeiro \\ C.P. 68528, Rio de Janeiro, 21941-972, RJ, Brazil
}

Received on 15 August, 2003.

\begin{abstract}
We investigate the onset of spinodal decomposition in a relativistic fluid of quarks coupled to a nonequilibrium chiral condensate. Studying small fluctuations around equilibrium, we identify the role played by sound and chiral waves in the generation of unstable modes.
\end{abstract}

The hadronization of the quark-gluon plasma (QGP) possibly produced in the early universe or in high-energy heavy-ion collisions may proceed in a number of different ways, depending on the nature of the QCD phase transition. In the heavy-ion case, some results from CERNSPS and BNL-RHIC suggest what has been called sudden hadronization [1] or explosive behavior [2, 3]. From the theoretical side, this phenomenon has been associated associated with deep supercooling of the QGP followed by spinodal decomposition, and also to rapid changes in the effective potential of QCD near the critical temperature, such as predicted, for instance, by the Polyakov loop model [4]. Clearly, an understanding of the interplay between the typical space and time scales of the expanding plasma is welcome. Some attempts in this direction can be found in Refs. [5, 6, 7, 8, 9, 10, 11, 12, 13].

In this paper, we discuss the onset of spinodal instability in an expanding plasma. As a phenomenological model to mimic the case of the QGP, we use a relativistic plasma of quarks coupled to a chiral field. The later is not necessarily in thermal equilibrium with the quark fluid. Although we derive a phenomenological nonequilibrium chiral hydrodynamics from a variational principle, we do not focus on the numerical solution of the resulting hydrodynamic transport equations.

To model the mechanism of chiral symmetry breaking present in QCD, we adopt a simple low-energy effective chiral model: the linear $\sigma$-model coupled to quarks [14], which in turn comprise the hydrodynamic degrees of freedom of the system. Similar approaches, relying on low-energy effective models for QCD and making use of a number of techniques to treat the expanding plasma, can be found in the literature $[5,6,7,8,9]$. The gas of quarks provides a thermal bath in which the long-wavelength modes of the chiral field evolve. The latter plays the role of an order parameter in a Landau-Ginzburg description of the chiral phase transition $[8,9]$.

Let us consider a chiral field $\phi=(\sigma, \vec{\pi})$, where $\sigma$ is a scalar field and $\pi^{i}$ are pseudoscalar fields playing the role of the pions, coupled to two flavors of quarks according to the
Lagrangian:

$$
\mathcal{L}=\bar{q}\left[i \gamma^{\mu} \partial_{\mu}+\mu_{q} \gamma^{0}-W(\phi)\right] q+\frac{1}{2} \partial_{\mu} \phi \partial^{\mu} \phi-V(\phi) .
$$

Here $q=(u, d)$ is the constituent-quark field and $\mu_{q}=\mu / 3$ is the quark chemical potential. The interaction between the quarks and the chiral field is given by $W(\phi)=g(\sigma+$ $\left.i \gamma_{5} \vec{\tau} \cdot \vec{\pi}\right)$, and $V(\phi)=\left(\lambda^{2} / 4\right)\left(\sigma^{2}+\vec{\pi}^{2}-v^{2}\right)^{2}-h_{q} \sigma$ is the self-interaction potential for $\phi$. The parameters above are chosen such that chiral $S U_{L}(2) \otimes S U_{R}(2)$ symmetry is spontaneously broken in the vacuum. The vacuum expectation values of the condensates are $\langle\sigma\rangle=f_{\pi}$ and $\langle\vec{\pi}\rangle=0$, where $f_{\pi}=93 \mathrm{MeV}$ is the pion decay constant. The explicit symmetry breaking term is due to the finite currentquark masses and is determined by the PCAC relation, giving $h_{q}=f_{\pi} m_{\pi}^{2}$, where $m_{\pi}=138 \mathrm{MeV}$ is the pion mass. This yields $v^{2}=f_{\pi}^{2}-m_{\pi}^{2} / \lambda^{2}$. The value of $\lambda^{2}=20$ leads to a $\sigma$-mass, $m_{\sigma}^{2}=2 \lambda^{2} f_{\pi}^{2}+m_{\pi}^{2}$, equal to $600 \mathrm{MeV}$. The quark-chiral field coupling constant is taken to be $g=3.3$, for which the constituent quark mass is $307 \mathrm{MeV}$, about 1/3 of the nucleon mass.

In what follows, we treat the gas of quarks as a heat bath for the chiral field, with temperature $T$ and baryon-chemical potential $\mu$. Integrating over the fermionic degrees of freedom and using a classical approximation for the chiral field, we can write the thermodynamic potential as

$$
\Omega(T, \mu, \phi)=V(\phi)-\frac{T}{\mathcal{V}} \ln \operatorname{det}\left\{\left[G_{E}^{-1}+W(\phi)\right] / T\right\},
$$

where $G_{E}$ is the fermionic Euclidean propagator and $\mathcal{V}$ is the (infinite) volume of the system. The determinant that results from the functional integration over the quark and anti-quark fields can be calculated to one-loop order in the standard fashion $[15,16]$. As already mentioned, the chiral field $\phi$ plays the role of an order parameter, its equilibrium value corresponding to the minimum of the grand canonical potential (2) for given $(T, \mu)$.

The collective modes of the quark heat bath will be treated within the framework of ideal relativistic hydrodynamics. To obtain the equations of motion we generalize the variational principle of Ref. [17], including the coupling of the hydrodynamical degrees of freedom to the chiral field 
dynamics. This approach provides a natural way of merging chiral and fluid dynamics in a unified Lagrangian system. For a different treatment of the hydrodynamics of nuclear matter in the chiral limit, see [18].

We describe the state of the fluid in terms of the fourvelocity $u^{\mu}(x)=(\gamma, \gamma \vec{v})$, where $\vec{v}(\vec{r}, t)$ is the flow velocity of matter, the proper baryon density, $n(x)$, and the proper entropy density, $s(x)$. The action of the fluid-chiral field system is then defined as

$$
S \equiv \int d^{4} x\left[\frac{1}{2} \partial_{\mu} \phi \partial^{\mu} \phi-\epsilon(n, s, \phi)\right],
$$

where $\epsilon=\Omega+T s+\mu n$ is the energy density, from which the temperature and chemical potential are obtained by the usual thermodynamic relations: $T=\partial \epsilon(n, s, \phi) / \partial s$ and $\mu=\partial \epsilon(n, s, \phi) / \partial n$. The variation of $n, s$ and $u^{\mu}$ in the action principle is performed under constraints arising from baryon number conservation, $\partial_{\mu}\left(n u^{\mu}\right)=0$, entropy conservation, $\partial_{\mu}\left(s u^{\mu}\right)=0$, and normalization of the 4-velocity, $u^{\mu} u_{\mu}=1$. With these conditions the variational principle, $\delta S=0$, leads to the equations of motion:

$$
\begin{gathered}
\square \phi=-R, \\
u^{\mu} \partial_{\mu}\left(w u^{\nu}\right)=-\left(\partial_{\mu} u^{\mu}\right) w u^{\nu}+\partial^{\nu} p+R \partial^{\nu} \phi,
\end{gathered}
$$

where $p=-\Omega$ is the pressure, $w=\epsilon+p=T s+\mu n$ is the enthalpy density, and

$$
R=\partial \Omega(T, \mu, \phi) / \partial \phi=\partial \epsilon(n, s, \phi) / \partial \phi
$$

gives the dynamical coupling between the fluid and the chiral field. Note that $R$ has four components, and can be written as

$$
R=\frac{\partial V(\phi)}{\partial \phi}+g \rho(T, \mu, \phi)
$$

where the (scalar/pseudoscalar) density $\rho$ is

$$
\rho=g \phi \nu_{q} \int \frac{d^{3} k}{(2 \pi)^{3}} \frac{1 / E_{k}(\phi)}{e^{\left[E_{k}(\phi)-\mu_{q}\right] / T}+1}+\left(\mu_{q} \rightarrow-\mu_{q}\right) .
$$

Here $\nu_{q}=12$ stands for the color-spin-isospin degeneracy of the quarks, $E_{k}(\phi)=\left(\vec{k}^{2}+m_{q}^{2}(\phi)\right)^{1 / 2}$, and $m_{q}(\phi)=$ $\left(g^{2} \phi^{2}\right)^{1 / 2}=g\left(\sigma^{2}+\vec{\pi}^{2}\right)^{1 / 2}$ plays the role of an effective mass for the quarks.

Equation (5) is the relativistic Euler equation for the quark fluid in the presence of the chiral field. Introducing the energy-momentum tensor of the fluid in the usual way, $T^{\mu \nu}=w u^{\mu} u^{\nu}-p g^{\mu \nu}$, we can write the Euler equation as

$$
\partial_{\mu} T^{\mu \nu}=R \partial^{\nu} \phi
$$

The total energy-momentum tensor of the fluid-field system, $\mathcal{T}^{\mu \nu}=T^{\mu \nu}-\frac{1}{2} \partial_{\alpha} \phi \partial^{\alpha} \phi g^{\mu \nu}+\partial^{\mu} \phi \partial^{\nu} \phi$, is, of course, conserved. Different derivations and equivalent forms of Eqs. (4)-(9) can be found in [5, 6, 9].

Numerical studies of the system above have been done extensively in Refs. [6, 9]. Here, we refrain from this approach and, instead, analyze the behavior of small perturbations around equilibrium. We write $\psi(x)=\psi_{e q}+\psi_{1} e^{-i K x}$, where $\psi$ stands for $n, s, \vec{v}$ or $\phi$, and $K^{\mu}=(\omega, \vec{k}) . \psi_{e q}$ corresponds to a situation of stable or metastable equilibrium, given by the equation $R=0$. Keeping terms up to first order in the perturbation $\psi_{1}$, the field and hydrodynamical equations become

$$
\begin{aligned}
\left(\omega^{2}-\vec{k}^{2}-m_{\pi}^{2}\right) \vec{\pi}_{1} & =0 \\
\left(\omega^{2}-\vec{k}^{2}-m_{\sigma}^{2}\right) \sigma_{1} & =\frac{k}{\omega} w_{e q} R^{\prime} v_{1}, \\
\left(\omega^{2}-p^{\prime} \vec{k}^{2}\right) v_{1} & =\omega k R^{\prime} \sigma_{1},
\end{aligned}
$$

where the masses of the $\sigma$ and $\vec{\pi}$ fields are $m_{\sigma}^{2}=$ $\left(\partial^{2} \epsilon(n, s, \phi) / \partial \sigma^{2}\right)_{e q}$ and $m_{\pi}^{2}=\left(\partial^{2} \epsilon(n, s, \phi) / \partial \pi^{2}\right)_{e q}$. We have also defined $p^{\prime} \equiv[\partial p / \partial \epsilon]_{e q}$ and $R^{\prime} \equiv\left[\partial R_{\sigma} / \partial \epsilon\right]_{e q}$, evaluated at constant $(s / n)$ and $\phi$. The flow velocity $\vec{v}_{1}$ is parallel to the wave vector $\vec{k}$ (sound waves are longitudinal), and its magnitude determines the baryon number and entropy amplitudes:

$$
\begin{gathered}
n_{1}=(k / \omega) n_{e q} v_{1}, \\
s_{1}=(k / \omega) s_{e q} v_{1} .
\end{gathered}
$$

We see from the linearized equations (10)-(12) that, to first order, only the $\sigma$ field is coupled to the hydrodynamic degrees of freedom. The dispersion relation for the coupled modes reads

$$
\left(\omega^{2}-p^{\prime} \vec{k}^{2}\right)\left(\omega^{2}-\vec{k}^{2}-m_{\sigma}^{2}\right)=w_{e q}{R^{\prime}}^{2} \vec{k}^{2}
$$

For long wavelength fluctuations we can approximate the roots of (15) by

$$
\begin{aligned}
\omega_{s}^{2} / \vec{k}^{2} & =p^{\prime}-\frac{M^{2}}{m_{\sigma}^{2}}+\mathcal{O}\left(\vec{k}^{2}\right) \\
\omega_{\sigma}^{2} & =m_{\sigma}^{2}+\mathcal{O}\left(\vec{k}^{2}\right)
\end{aligned}
$$

where $M^{2} \equiv w_{e q} R^{\prime 2}$. These modes can be identified as sound waves (frequency $\omega_{s}$ ) and chiral waves (frequency $\left.\omega_{\sigma}\right)$.

The onset of instabilities takes place when $\omega^{2}<0$, i.e., either for $\left(p^{\prime}-M^{2} / m_{\sigma}^{2}\right)<0$ or for $m_{\sigma}^{2}<0$. From Eq. (16), however, we see that sound waves become unstable before chiral waves do.

Let us now discuss the onset of this spinodal instability in different portions of the phase diagram of the linear $\sigma$-model coupled to quarks. 


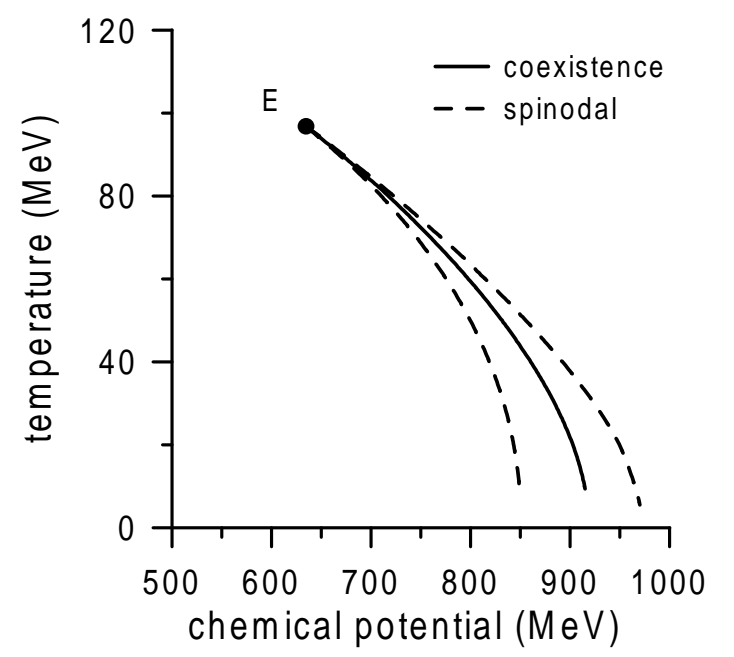

Figure 1. Phase diagram for the effective model in the $(\mu, T)$ plane. The coexistence curve ending at the critical point $E$ is represented by the solid line. The spinodal curves are shown as dashed lines.

In Fig. 1 we plot the phase diagram in the $(\mu, T)$-plane. The coexistence curve is represented by the solid line. It ends at a critical point, $E$, located at $T_{E}=98 \mathrm{MeV}$ and $\mu_{E}=630 \mathrm{MeV}$. The spinodal lines $\left(p^{\prime}-M^{2} / m_{\sigma}^{2}=0\right)$ corresponding to supercooling and superheating are shown as dashed lines.

A more illuminating picture of the unstable region is given by the phase diagram in the $(n, T)$-plane, as shown in Fig. 2. As before, the phase coexisting border is represented by the solid line and dashed lines stand for the spinodals. The sector on the right of the critical point $E$ corresponds to supercooled states in the chirally symmetric phase. The domain inside the dashed lines corresponds to the spinodal unstable states.

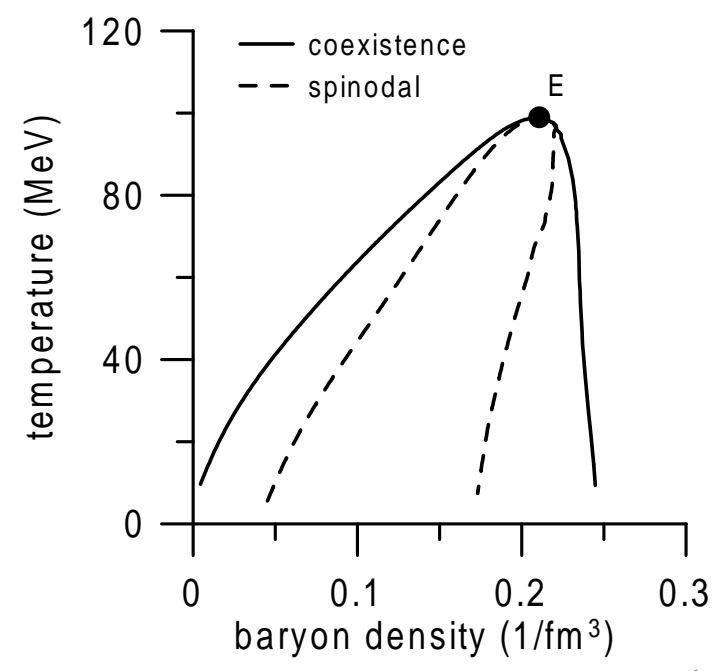

Figure 2. Phase diagram for the effective model in the $(n, T)$ plane. Line conventions are the same as in Fig. 1.

To summarize, we have shown that spinodal decomposition in the QGP proceeds by the exponential increase of hydrodynamical sound-like modes. We have mapped the boundaries of the unstable region, determining how much supercooling (or superheating) is necessary to trigger spinodal instability. In the case of relativistic heavy ion reactions, the rapid time scale of expansion and effects due to the finite size of the system could change significantly these results. In order to investigate these effects in a realistic and quantitative way, one should perform numerical simulations of the evolution of the instability in different scenarios. In particular, one should study the limits of the linear approximation at large times. Results in this direction will be presented elsewhere.

Acknowledgments This work was partially supported by CAPES, CNPq, FAPERJ and FUJB/UFRJ.

\section{References}

[1] J. Rafelski and J. Letessier, Phys. Rev. Lett. 85, 4695 (2000).

[2] A. Dumitru and R. D. Pisarski, Nucl. Phys. A 698, 444 (2002).

[3] O. Scavenius, A. Dumitru and A. D. Jackson, Phys. Rev. Lett. 87, 182302 (2001).

[4] R. D. Pisarski, Phys. Rev. D 62, 111501 (2000); A. Dumitru and R. D. Pisarski, Phys. Lett. B 504, 282 (2001).

[5] L. P. Csernai and I. N. Mishustin, Phys. Rev. Lett. 74, 5005 (1995); A. Abada and J. Aichelin, Phys. Rev. Lett. 74, 3130 (1995); A. Abada and M. C. Birse, Phys. Rev. D 55, 6887 (1997).

[6] I. N. Mishustin and O. Scavenius, Phys. Rev. Lett. 83, 3134 (1999).

[7] O. Scavenius and A. Dumitru, Phys. Rev. Lett. 83, 4697 (1999).

[8] O. Scavenius, A. Dumitru, E. S. Fraga, J. T. Lenaghan and A. D. Jackson, Phys. Rev. D 63, 116003 (2001).

[9] K. Paech, H. Stöcker and A. Dumitru, nucl-th/0302013.

[10] E. E. Zabrodin, L. V. Bravina, L. P. Csernai, H. Stöcker and W. Greiner, Phys. Lett. B 423, 373 (1998).

[11] P. Shukla and A. K. Mohanty, Phys. Rev. C 64, 054910 (2001).

[12] C. Spieles, H. Stöcker and C. Greiner, Phys. Rev. C 57, 908 (1998).

[13] E. S. Fraga and R. Venugopalan, arXiv:hep-ph/0304094.

[14] M. Gell-Mann and M. Levy, Nuovo Cim. 16, 705 (1960); R. D. Pisarski, Phys. Rev. Lett. 76, 3084 (1996).

[15] O. Scavenius, A. Mocsy, I. N. Mishustin and D. H. Rischke, Phys. Rev. C 64, 045202 (2001).

[16] J. Kapusta, Finite Temperature Field Theory (Cambridge University Press, Cambridge, 1989).

[17] H. T. Elze, Y. Hama, T. Kodama, M. Makler and J. Rafelski, J. Phys. G 25, 1935 (1999).

[18] D. T. Son, Phys. Rev. Lett. 84, 3771 (2000). 